\title{
All-sky search for correlations in the arrival directions of astrophysical neutrino candidates and ultrahigh-energy cosmic rays
}

\author{
The IceCube Collaboration ${ }^{1}$, The Pierre Auger Collaboration ${ }^{2}$, The Telescope Array \\ Collaboration ${ }^{3}$ \\ ${ }^{1}$ http://icecube.wisc.edu/collaboration/authors/icrc17_icecube \\ 2 http://www.auger.org/archive/authors_icrc_2017.html \\ ${ }^{3}$ http://www.telescopearray.org/index.php/research/collaborators
}

E-mail: imen.alsamarai@icecube.wisc.edu; golupg@cab.cnea.gov.ar

\begin{abstract}
High-energy neutrinos, being neutral and weakly interacting particles, are powerful probes of the sites of production and acceleration of cosmic rays. The challenging discovery of cosmic neutrinos by the IceCube Collaboration has moved the field closer to realizing the potential of neutrino astronomy. Meanwhile, ground-based cosmic ray detectors like the Pierre Auger Observatory and the Telescope Array have reached an unprecedented accuracy in the determination of the features of the cosmic rays at the highest energies. We report on a collaborative effort between IceCube, the Pierre Auger Observatory and Telescope Array to identify directional correlations between the arrival directions of the highest-energy cosmic rays from both hemispheres and of the most probable cosmic neutrino events detected by IceCube. We describe the updated results of two independent searches using seven years of IceCube neutrino data and the most energetic cosmicray events detected by the Pierre Auger Observatory and the Telescope Array. The directional correlation found between UHECRs and neutrinos is reported with a significance of $\sim 2 \sigma$.
\end{abstract}

Corresponding authors: G. Golup ${ }^{4}$, I. Al Samarai* ${ }^{5}$, L. Caccianiga ${ }^{6}$, A. Christov ${ }^{5}$, P.L. Ghia ${ }^{7}$, U. Giaccari ${ }^{8}$, T. Montaruli ${ }^{5}$, H. Sagawa ${ }^{9}$, L. Schumacher ${ }^{10}$, P. Tinyakov ${ }^{11}$.

${ }^{4}$ Centro Atómico Bariloche, S.C de Bariloche, Argentina

5 Départment de physique nucléaire et corpusculaire, Université de Genève, Genève, Switzerland.

${ }^{6}$ Università di Milano, Dipartimento di Fisica, Italy

7 Institut de Physique Nucléaire d'Orsay (IPNO), Université Paris-Sud, Univ. Paris/Saclay, CNRS-IN2P3, France

${ }^{8}$ Universidade Federal do Rio de Janeiro, Instituto de Fisíca, Rio de Janeiro, Brazil

${ }^{9}$ Institute for Cosmic Ray Research, University of Tokyo, Kashiwa, Chiba, Japan.

${ }^{10}$ RWTH Aachen University, III. Physikalisches Institut B, Aachen, Germany.

${ }^{11}$ Service de Physique Théorique, Université Libre de Bruxelles, Ixelles, Belgium.

35th International Cosmic Ray Conference ICRC 2017-

10-20 July, 2017

Bexco, Busan, Korea

* Speaker. 


\section{Introduction}

The determination of the origin of cosmic rays (CRs) is a difficult task since CRs are deflected during propagation. The extent of this angular deflection is still poorly constrained. Neutrinos produced during hadronic interactions of $\mathrm{CR}$ primaries propagate unaffected from their sources to us. They can therefore deliver potentially valuable information on the most energetic CR sources of the Universe. Neither of the observatories of neutrinos or ultrahigh-energy cosmic rays (UHECRs) dedicated to unravel the quest of the CR origin have delivered evidence for specific cosmic sources. This search for a common origin of UHECRs and neutrinos results from a joint collaboration between the IceCube Neutrino Observatory, the Pierre Auger Observatory and the Telescope Array (TA). Previous results of this work [1] provided a potentially interesting connection between neutrino and UHECR directions at the $3 \sigma$ level. We update that work here including two additional years of neutrino data from IceCube and one more year of TA data.

\section{The observatories and the data sets}

\section{The IceCube South Pole Neutrino Observatory}

IceCube [2] is a cubic-kilometer high-energy neutrino detector (energy threshold $>\sim 100 \mathrm{GeV}$ ) located at the geographic South Pole at about 1.5 to $2.5 \mathrm{~km}$ beneath the ice sheet surface. It is composed of 86 strings instrumented by 5160 photomultiplier tubes housed in pressure resistant spheres. Since 2005, data have been taken with partially completed configurations (9, 22, 40, 59 and 79 strings) until its final completion in December 2010. In 2013, a neutrino flux compatible with astrophysical neutrino expectations was reported $[3,4,5]$. Cumulating four years of data, the hypothesis of a pure atmospheric origin has been rejected at $\sim 6.5 \sigma$ level. These detected neutrinos are of all flavors interacting inside the detection volume (starting events) with deposited energies ranging from $60 \mathrm{TeV}$ up to $2 \mathrm{PeV}$. They compose the HESE dataset ('High-Energy Starting Events'). They are mostly composed of shower-like events (cascades) characterized by an angular resolution of $\sim 15^{\circ}$ above $100 \mathrm{TeV}$. The track-like events are induced by muons and have a better angular resolution of $\lesssim 1^{\circ}$. The resolution of the deposited energy for tracks and cascades is around $15 \%$ [6] but cascades have a better resolution for the reconstructed neutrino energy since most of the energy is deposited in the detector, which is not the case for tracks.

In this analysis, we present results on the published updated sample of 39 HESE cascades and 7 HESE tracks [1] with two additional years of data composed of 19 cascades and 8 tracks, giving a total of 58 cascades and 15 tracks constituting the six-year HESE dataset [7].

A complementary dataset of through-going muons induced by charged current interactions of candidate $v_{\mu}$ from the Northern sky [8] is also added to the track dataset. It has been reported that events giving this set of tracks do have a spectrum that is inconsistent with the hypothesis of

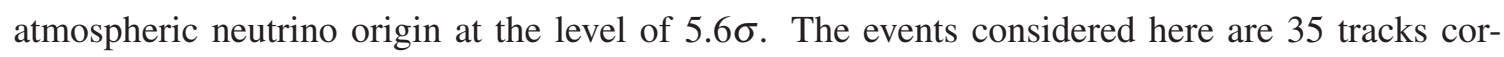
responding to seven years of data from the eight-year data sample presented in [9]. Only events with 'signalness' $>50 \%$ are considered, where the signalness is defined as the ratio of the astrophysical expectation over the sum of the atmospheric and astrophysical expectations for a given energy proxy and best-fit neutrino spectral index of 2.16 . This requirement of 'signalness' $>50 \%$ 
translates to a selection of events with a lower energy threshold of $\sim 200 \mathrm{TeV}$ of the muon energy proxy [8].

The events from the HESE sample and that from the complementary dataset from the Northern sky only confirm the picture of an isotropic neutrino emission, but measured differences in the energy spectrum may suggest a mixed origin of the events detected in IceCube. Nonetheless, no astrophysical counterpart has so far been supported by the current observational data.

\section{The Pierre Auger Observatory}

The Pierre Auger Observatory [10] is a hybrid high-energy cosmic-ray detector, covering an area of about $3000 \mathrm{~km}^{2}$, located in Argentina. It combines a large surface detector array (SD) composed of 1660 water-Cherenkov detectors with an atmospheric fluorescence detector (FD) made of 27 fluorescence telescopes. The dataset used for this analysis is composed of 231 cosmic rays with energies $\mathrm{E}_{C R} \geq 52 \mathrm{EeV}$ recorded with the SD array from January 2004 to March 2014. The cut on the zenith angle $\theta \leq 80^{\circ}$ allows for a field-of-view ranging from $-90^{\circ}$ to $+45^{\circ}$ in declination. The angular resolution, defined as the $68^{\text {th }}$ percentile of the distribution of the opening angles between the true and reconstructed directions of simulated events, is better than $0.9^{\circ}$ [11].

\section{Telescope Array}

Telescope Array (TA) is a $700 \mathrm{~km}^{2}$ cosmic-ray surface array detector located in the United States [12]. It is composed of 507 plastic scintillators distributed on a square grid with $1.2 \mathrm{~km}$ spacing overlooked by three fluorescence detector stations housing 38 telescopes. The selected events have an energy $E_{\mathrm{CR}} \geq 57 \mathrm{EeV}$ and zenith angles smaller than $55^{\circ}$. The angular resolution of these events is about $1.5^{\circ}$. In addition to the 87 events detected from May 2008 to May 2014 and used in [1], 22 additional events collected in an additional year of data were used, reaching a total of 109 UHECRs from TA.

In both UHECR observatories, the absolute energy scale is given by fluorescence calibrations, using 'hybrid' events which are detected simultaneously by the SD array and the FD. For these events, it is possible to take advantage of the quasi-calorimetric energy determination from the FD technique. For the Pierre Auger Observatory, the systematic uncertainty on the energy scale is $14 \%$ and the statistical energy uncertainty is smaller than $12 \%$ [13]. For TA, the energy resolution is $\sim 20 \%$, while the systematic uncertainty on the energy scale is $22 \%$ [14]. In accordance with the TA and Auger Energy Spectrum Working Group, and as presented in [1], the energy measured by TA is downshifted by $13 \%$, so that the measured Auger and TA energy spectra coincide at $10 \mathrm{EeV}$.

The directions of the neutrinos detected by IceCube and the UHECRs detected by the Pierre Auger Observatory and Telescope Array used in this work are shown in Figure 1 in Equatorial coordinates. The complementary field-of-views of the UHECR observatories allow for an all-sky search for correlations with neutrinos of all-flavors detected by IceCube.

\section{The methods}

The search for correlations in the arrival directions of UHECRs and neutrinos relies on two in- 


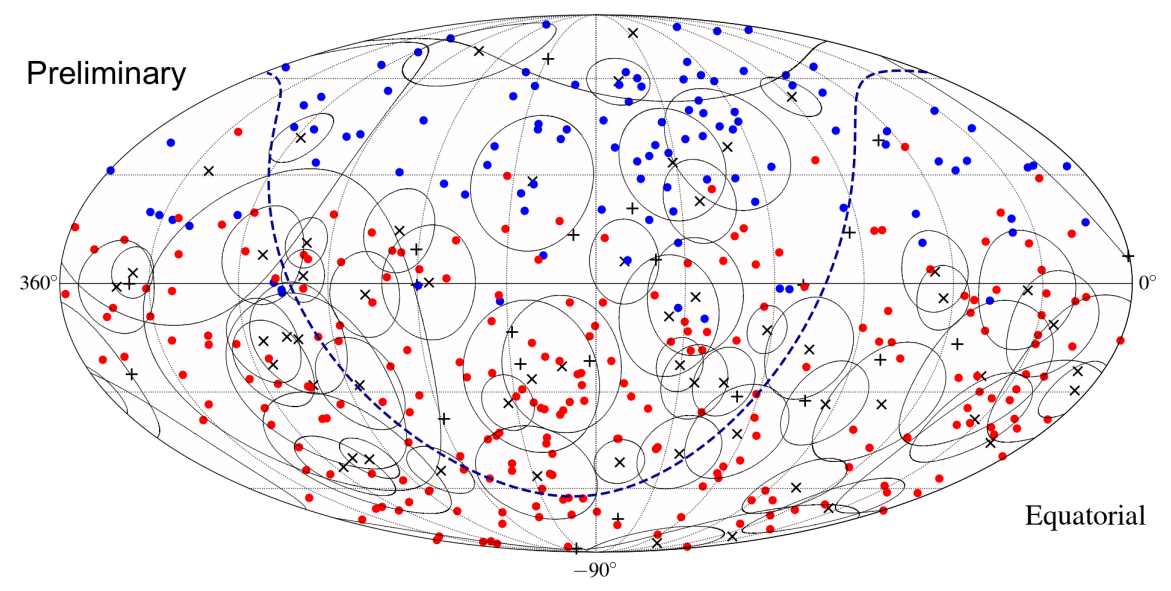

Figure 1: Directions of the UHECR events detected by Telescope Array (blue points) and the Pierre Auger Observatory (red points). The directions of the shower-like neutrino events detected by IceCube are shown in black crosses surrounded by the angular uncertainties shown in black circles. The track-like events are shown with 'plus' signs. The Galactic plane is represented by the dashed blue line.

dependent methods adapted for this analysis [1]: the unbinned-likelihood and the cross-correlation method.

\section{Cross-correlation method}

The cross-correlation method consists in computing the relative excess in the number of neutrino-UHECR pairs as a function of their angular separation over the expectation of isotropically distributed CR arrival directions, keeping the arrival directions of the neutrinos fixed. The isotropic distribution of the arrival directions of UHECRs is simulated according to the corresponding geometric exposures of the observatories. We also compare the number of pairs to an isotropic distribution of neutrinos, keeping the arrival directions of the UHECRs fixed and thus preserving the degree of anisotropy in the arrival directions of CRs. The isotropic flux of neutrinos is simulated by producing random right ascensions and keeping their declination fixed to account for the declination dependence in the IceCube acceptance. The angular separation in this study ranges from $1^{\circ}$ to $30^{\circ}$ with steps of $1^{\circ}$. This angular scan does not require one to make an assumption on the deflection of CRs while they propagate from their (supposedly) common source with neutrinos.

\section{The unbinned-likelihood method}

The second test is a stacking likelihood test assuming that the stacked sources are the neutrino directions. This test requires a hypothesis on the CR deflections. We have nonetheless made a scan on different values of the deflections also to account for the uncertainty on the composition of the CRs.

We considered a few models of cosmic ray deflections, which are based on backtracking simulations of UHECRs in the Galactic magnetic field models of Pshirkov et al. [15] and Jansson and Farrar [16]. Assuming a pure proton composition with an energy $E_{\mathrm{CR}}=100 \mathrm{EeV}$, we obtained a median angular deflection of $2.7^{\circ}$ due to the Galactic magnetic field. In this work, the assumed 
angular deflections in the $\mathrm{CR}$ directions are thus taken as $3^{\circ} \times 100 \mathrm{EeV} / E_{\mathrm{CR}}$. To account for a possible heavier composition or larger contribution of the intervening magnetic fields, additional test values of $6^{\circ} \times 100 \mathrm{EeV} / E_{\mathrm{CR}}$ and $9^{\circ} \times 100 \mathrm{EeV} / E_{\mathrm{CR}}$ were considered. It is to be noted that the likelihood test is less optimal but not insensitive if the 'true' deflection of CRs is slightly different than foreseen by models. The expression of the log-likelihood is defined as:

$$
\ln \mathscr{L}\left(n_{\mathrm{s}}\right)=\sum_{i=1}^{N_{\text {Auger }}} \ln \left(\frac{n_{\mathrm{s}}}{N_{\mathrm{CR}}} S_{\text {Auger }}^{i}+\frac{N_{\mathrm{CR}}-n_{\mathrm{s}}}{N_{\mathrm{CR}}} B_{\text {Auger }}^{i}\right)+\sum_{i=1}^{N_{\mathrm{TA}}} \ln \left(\frac{n_{\mathrm{s}}}{N_{\mathrm{CR}}} S_{\mathrm{TA}}^{i}+\frac{N_{\mathrm{CR}}-n_{\mathrm{s}}}{N_{\mathrm{CR}}} B_{\mathrm{TA}}^{i}\right),
$$

where $n_{\mathrm{s}}$, the number of signal events, is the only free parameter, $N_{\mathrm{CR}}=N_{\mathrm{Auger}}+N_{\mathrm{TA}}$ is the total number of UHECR events (340), $S_{\text {Auger }}^{i}$ and $S_{\text {TA }}^{i}$ are the signal PDFs (Probability Distribution Functions) for Auger and for TA, respectively, and $B_{\text {Auger }}^{i}$ and $B_{\mathrm{TA}}^{i}$ are the corresponding background PDFs. The signal PDFs, in which the different neutrino positions are stacked, take into account the exposure and angular resolution of the CR observatories, the assumed CR magnetic deflections and the likelihood maps for the reconstruction of the $v$ arrival directions (Figure 2). Thus, for each $\mathrm{CR} i$ at a given direction $\vec{r}_{i}$ and energy $E_{i}$, the signal PDF is expressed as:

$$
S_{\text {CR observatory }}^{i}\left(\vec{r}_{i}, E_{i}\right)=R_{\mathrm{CR} \text { observatory }}\left(\delta_{i}\right) \cdot \sum_{j=1}^{N_{s r c}} S_{j}\left(\vec{r}_{i}, \sigma\left(E_{i}\right)\right)
$$

$R_{\mathrm{CR} \text { observatory }}\left(\delta_{i}\right)$ is the relative exposure for a given event declination and $N_{s r c}$ is the number of stacked sources, 58 for the cascades and 49 for the tracks. The last term, $S_{j}\left(\vec{r}_{i}, \sigma\left(E_{i}\right)\right)$, is the value of the normalized directional likelihood map for the $j^{\text {th }}$ source (i.e neutrino) taken at $\vec{r}_{i}$ and smeared with a Gaussian with standard deviation $\sigma\left(E_{i}\right)$. The Gaussian smearing takes into account the energy-dependent magnetic deflections imprint on the CR directions $\sigma\left(E_{i}\right)=$ $\sqrt{\sigma_{\mathrm{CR} \text { observatory }}^{2}+\sigma_{M D}^{2}}$, where $\sigma_{\mathrm{CR} \text { observatory }}$ is the angular resolution of the $\mathrm{CR}$ observatory $\left(0.9^{\circ}\right.$ for Auger and $1.5^{\circ}$ for TA) and $\sigma_{M D}=D \times 100 \mathrm{EeV} / E_{\mathrm{CR}}$. Figure 2 represents the directional likelihood maps of the stacked neutrinos for shower-like and track-like topologies before smearing and convolved with the exposure of each CR observatory. The background PDFs are obtained from the normalized (by the total number of detected events by each observatory) exposures of the CR observatories. The test statistic $T S$ is defined as: $T S=-2 \ln \frac{\mathscr{L}\left(n_{\mathrm{s}}\right)}{\mathscr{L}\left(n_{\mathrm{s}}=0\right)}$ and follows a distribution close to $\chi^{2}$ with one degree of freedom.

\section{Results}

\section{Cross-correlation results}

Applying the cross-correlation method to the data, it is found that the maximum departure from the expectation for an isotropic CR flux, keeping the arrival directions of the neutrinos fixed, occurs at an angular distance of $1^{\circ}$ for tracks and $22^{\circ}$ for cascades, with post-trial $p$-values of 0.48 and $5.4 \times 10^{-3}$, respectively. In Figure 3, the relative excess of neutrino-UHECR pairs found in the data, $\left[n_{\mathrm{p}}(\alpha) /\left\langle n_{\mathrm{p}}^{\text {iso }}(\alpha)\right\rangle\right]-1$, as a function of the scanned separation angles is shown with respect to the expectations of an isotropic flux of CRs. Evaluating the significance under the hypothesis of an isotropic flux of neutrinos, keeping the arrival directions of the CRs fixed, we find that for the high-energy cascades the maximum departure from isotropic expectations is at $16^{\circ}$, with a post-trial $p$-value of $1.0 \times 10^{-2}$. 

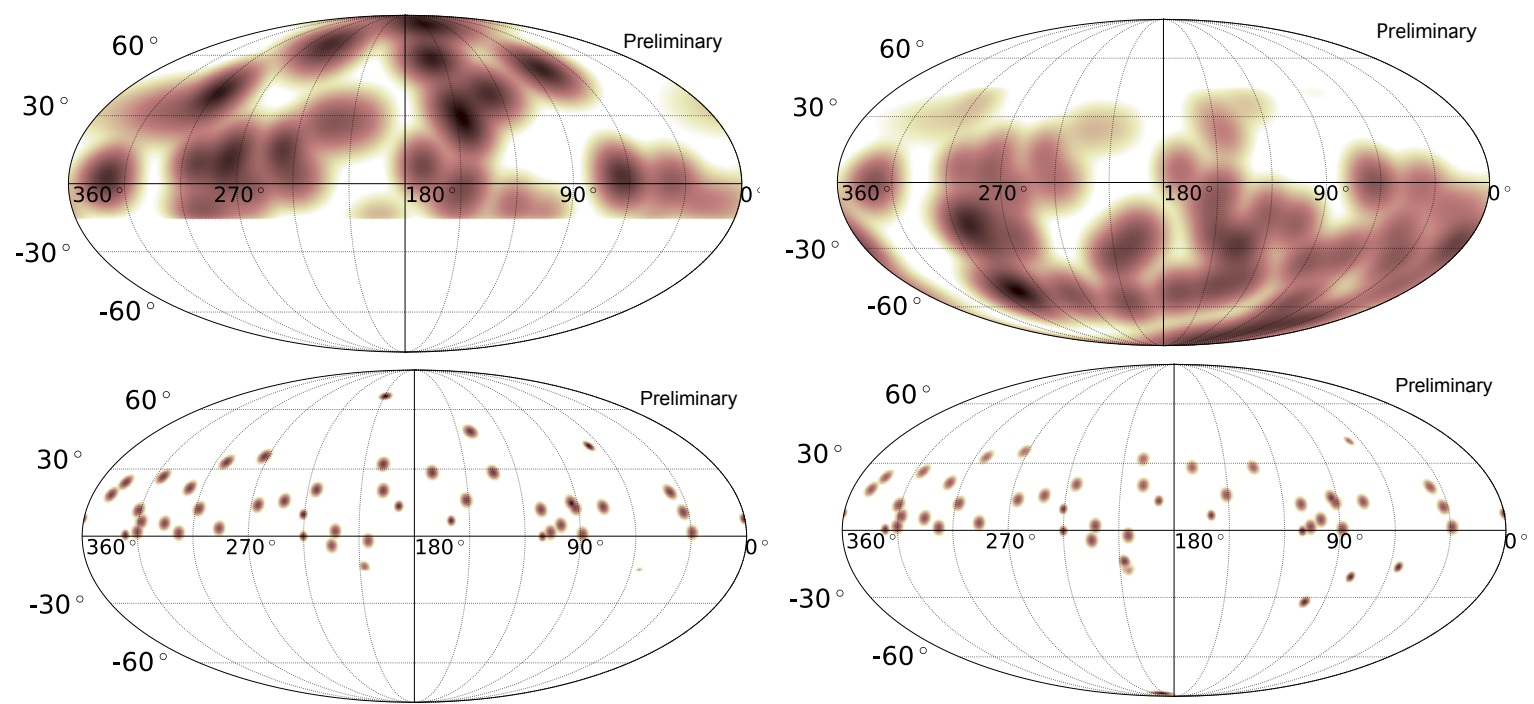

Figure 2: The signal PDFs before the Gaussian smearing in equatorial coordinates. The upper plots are for the high-energy cascades, while the lower ones are for the high-energy tracks. The declination-dependent exposure is applied for Auger in the right-hand plots and for TA in the left-hand plots.
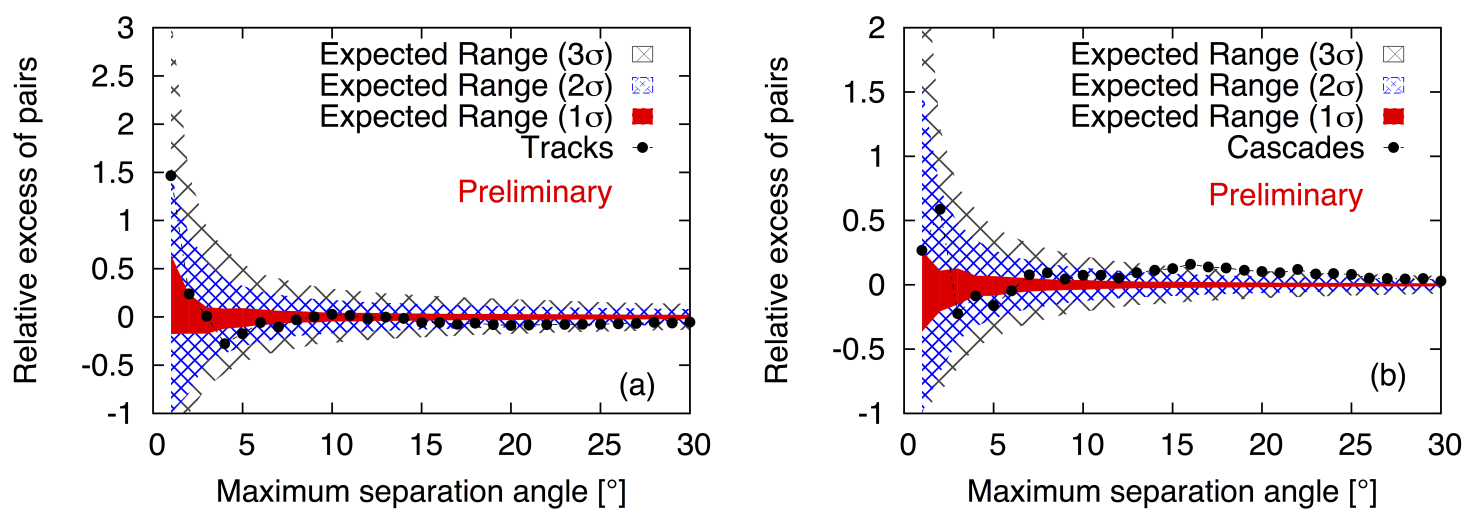

Figure 3: Relative excess of pairs, $\left[n_{\mathrm{p}}(\alpha) /\left\langle n_{\mathrm{p}}^{\text {iso }}(\alpha)\right\rangle\right]-1$, as a function of the maximum angular separation between the neutrino and UHECR pairs, for the analysis done with the high-energy tracks (left) and with the high-energy cascades (right). The $1 \sigma, 2 \sigma$ and $3 \sigma$ fluctuations expected from an isotropic distribution of arrival directions of CRs, keeping the arrival directions of the neutrinos fixed, are shown in red, blue and grey, respectively.

\section{Unbinned likelihood method results}

The results for the stacking method are shown in Table 1. The most significant deviation from an isotropic flux of CRs occurs for the magnetic deflection parameter $D=6^{\circ}$ with the high-energy cascades. The observed pre-trial $p$-value of $1.0 \times 10^{-2}$ corresponds to $2.2 \times 10^{-2}$ post-trial, by considering 1000 realizations of randomly distributed CRs with $6^{\circ} \times 100 \mathrm{EeV} / E_{\mathrm{CR}}$ deviation from the neutrino source positions. The test maintaining the CR directions fixed while simulating an 


\begin{tabular}{lllll}
\hline$D$ & \multicolumn{2}{c}{ High-energy tracks } & \multicolumn{2}{c}{ High-energy cascades } \\
& $n_{\mathrm{s}}$ & pre-trial $p$-value & $n_{\mathrm{s}}$ & pre-trial $p$-value \\
\hline $3^{\circ}$ & 0.9 & 0.44 & 45.5 & $2.7 \times 10^{-2}$ \\
$6^{\circ}$ & - & underfluctuation & 71.5 & $1.0 \times 10^{-2}$ \\
$9^{\circ}$ & - & underfluctuation & 84.7 & $1.5 \times 10^{-2}$ \\
\hline
\end{tabular}

Table 1: Results for the stacking analyses with the sample of high-energy tracks and high-energy cascades assuming an isotropic flux of CRs.

isotropic flux of neutrinos results in a post-trial $p$-value of $1.7 \times 10^{-2}$ for shower-like events.

\section{Discussion}

In [1], post-trial $p$-values of $2.7 \times 10^{-4}$ and $5 \times 10^{-4}$ with respect to an isotropic flux of UHECRs were found using the unbinned likelihood method and the cross-correlation method, respectively. With the addition of two years of HESE shower-like events, the updated $p$-values do not strengthen the hint of a possible anisotropic distribution of UHECRs and neutrinos previously found. Similarly, the computation of the $p$-values, assuming an isotropic flux of neutrinos while keeping the UHECR directions fixed, resulted in $p$-values which are less significant than those found in [1]. To illustrate the results found in this update, the UHECRs weights assuming $D=6^{\circ}$ and contributing to the signal PDF derived from equation 2.1 are shown in Figure 4 on top the neutrino directional maps in the exposures of the Auger Observatory and TA. Local clustering in the directions illustrate the $\sim 2.3 \sigma$ level correlation found.

It is noteworthy that this result can be explained by many facts. First, the not-yet-exhaustive knowledge of the CRs composition at such high energies and the poor knowledge of the Galactic magnetic field are the main limitations to the determination of the cosmic-ray sources using UHECRs. As already noted in [1], UHECRs can reach us from sources within the GZK horizon, meaning order of 10-100 Mpc. On the other hand, neutrinos can reach us from cosmological distances, so that if sources were stationary and uniformly distributed, only a few percent of neutrinos would be expected from the emitters of the detected UHECRs.

Many speculations on the possible sources of the four-year HESE sample still have not yet reached the significance of an evidence. The addition of two more years requires further correlation tests with various hypotheses of source candidates. It is also possible that there could be a contribution from galactic sources to the observed cosmic neutrino flux which would not be UHECR sources.

The future evolution of this search will concern the treatment of the magnetic deflection with updated models, the addition of updated data samples from the Pierre Auger Observatory and the cross-correlation with a sample of neutrinos of lower energy by IceCube and ANTARES.

\section{References}

[1] The IceCube, Pierre Auger and Telescope Array Collaborations, JCAP 1601 (2016) 037.

[2] IceCube Collaboration, Astropart. Phys. 26 (2006) 155. 

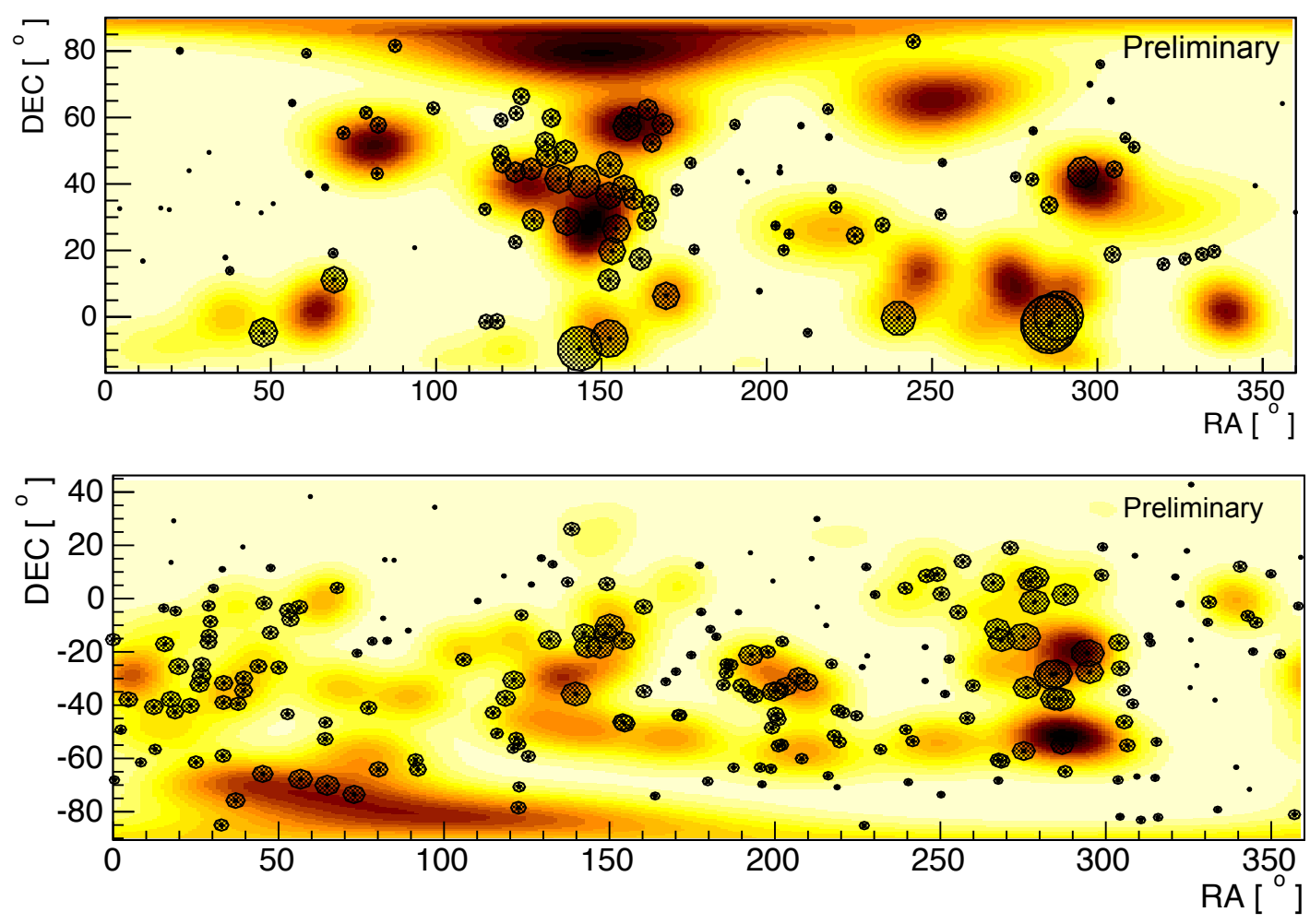

Figure 4: Neutrino signal PDF in the Telescope Array (top) and the Pierre Auger Observatory (bottom) exposures. The black dots represent the UHECRs directions. The black dashed circles radii are proportional to the weight assigned to each UHECR.

[3] IceCube Collaboration, Science 342 (2013) 1242856.

[4] IceCube Collaboration, Phys. Rev. Lett. 113 (2014) 101101.

[5] IceCube Collaboration, POS (ICRC2015) 1099 (2016).

[6] IceCube Collaboration, JINST 9 (2014) P03009.

[7] IceCube Collaboration, PoS (ICRC2017) 981 (these proceedings).

[8] IceCube Collaboration, Astrophys. J. 833 (2016) 3.

[9] IceCube Collaboration, POS (ICRC2 017) 1005 (these proceedings).

[10] The Pierre Auger Collaboration, Nucl. Instrum. Meth. A A798 (2015) 172.

[11] C. Bonifazi for the Pierre Auger Collaboration, Nucl. Phys. B (Proc. Suppl.) 190 (2009) 20.

[12] The Telescope Array Collaboration, Nucl. Instrum. Meth. A 689 (2012) 87 and Nucl. Instrum. Meth. A 676 (2012) 54.

[13] The Pierre Auger Collaboration, JCAP 8 (2014) 19; R. Pesce for the Pierre Auger Collaboration, Proc. 32nd ICRC, Beijing, China, 2 (2011) 214 [arXiv:1107.4809].

[14] The Telescope Array Collaboration, Astrophys. J. 768 (2013) L1.

[15] M. S. Pshirkov, P. G. Tinyakov, P. P. Kronberg, K. J. Newton-McGee, Astrophys. J. 738 (2011) 192.

[16] R. Jansson and G. R. Farrar, Astrophys. J. 757 (2012) 14. 